\title{
Short-term calorie restriction activates SIRT1-4 and -7 in cardiomyocytes in vivo and in vitro
}

\author{
WEI YU ${ }^{1 *}$, HUI-FEN ZHOU ${ }^{1,2^{*}}$, RUI-BO LIN ${ }^{1}$, YU-CAI FU ${ }^{3}$ and WEI WANG ${ }^{1}$ \\ ${ }^{1}$ Department of Cardiology, The Second Affiliated Hospital of Shantou University Medical College, Shantou, Guangdong 515041; \\ ${ }^{2}$ Department of Intensive Care Unit, Xiaolan Hospital Affiliated to Southern Medical University, \\ Zhongshan, Guangdong 528415; ${ }^{3}$ Laboratory of Cell Senescence, Shantou University \\ Medical College, Shantou, Guangdong 515041, P.R. China
}

Received July 6, 2013; Accepted January 28, 2014

DOI: $10.3892 / \mathrm{mmr} .2014 .1944$

\begin{abstract}
Calorie restriction (CR) has been shown to increase longevity and mitigate age-associated diseases in various organisms. Numerous studies have identified that sirtuin 1 (SIRT1) is upregulated by CR. However, the expression of SIRT isoforms 2-7 in response to CR in cardiomyocytes has yet to be elucidated. Therefore, the present study aimed to investigate the cellular localization and expression of SIRT1-7 in cardiomyocytes. Twenty SD rats were fed either ad libitum (AL) or a CR diet (60\% of AL) for three weeks. In addition, H9c2 cells were cultured in Dulbecco's modified Eagle medium (DMEM) supplemented with either normal- $(4.5 \mathrm{~g} / \mathrm{l})$ or low- (1 g/l) glucose concentrations for $24 \mathrm{~h}$, representing control or CR cells, respectively. CR rats were observed to have significantly lower heart and body weights (BWs) than control rats. Moreover, immunohistochemical analyzes revealed that SIRT1, 3-5 and 7 demonstrated similar localization patterns in $\mathrm{H} 9 \mathrm{c} 2$ cells and rat cardiac tissues, with SIRT1 and 7 predominantly located in the nucleus and SIRT3-5 in the cytoplasm. This was in contrast with SIRT2, which was detected exclusively in the cytoplasm in rat cardiac tissues, but was found predominantly in the nucleus in $\mathrm{H} 9 \mathrm{c} 2$ cells. The converse was observed for
\end{abstract}

Correspondence to: Professor Wei Wang, Department of Cardiology, The Second Affiliated Hospital of Shantou University Medical College, 69 Dong Xia North Road, Shantou, Guangdong 515041, P.R. China

E-mail: wangwei_sumc@126.com

*Contributed equally

Abbreviations: AL, ad libitum; BW, body weight; CR, calorie restriction; CVDs, cardiovascular diseases; DAB, 3-3'-diaminobenzidine; DMEM, Dulbecco's modified Eagle medium; ECL, enhanced chemoluminescence; FBS, fetal bovine serum; FoxOs, Forkhead transcription factors; NAD, nicotinamide adenine dinucleotide; qPCR, quantitative polymerase chain reaction; $\mathrm{SD}$, Sprague Dawley; Sir2, silent information regulator 2

Key words: calorie restriction, longevity, sirtuins, cardiomyocytes, in vivo, in vitro
SIRT6. Quantitative polymerase chain reaction revealed that the mRNA expression of SIRT1-4 and -7 was increased in the CR group. Western blot analysis further revealed that the protein expression of SIRT1-4 and -7 was significantly increased in the cardiac tissues of rats in the CR group. These results suggest that $\mathrm{CR}$ may attenuate age-associated changes through reducing BW. Moreover, short-term CR may activate SIRT1 as well as SIRT2-4 and -7 expression in cardiomyocytes in vivo and in vitro.

\section{Introduction.}

The association between calorie restriction (CR) and longevity was initially identified in a study by McCay et al (1), who observed that laboratory rodents maintained on a CR diet exhibited an increase in lifespan. CR has since become an area of extensive investigation. Numerous studies have shown that $\mathrm{CR}$ is capable of significantly increasing the lifespan in a range of organisms, from yeast to mammals $(2,3)$. Furthermore, marked physiological changes have been observed in CR rodents, including decreases in blood glucose, insulin levels and body weight (BW), and increases in insulin sensitivity, which have been suggested to be beneficial with regard to preventing the onset of cardiovascular diseases (CVDs) $(3,4)$. Futhermore, it has been suggested that CR may directly protect cardiomyocytes by reducing the levels of reactive oxygen species and thereby attenuating the development of atherosclerosis (5).

An enhanced understanding of CR may provide novel approaches for the treatment of CVD. A previous study suggested that some of the changes induced by CR require silent information regulator 2 (Sir2), and that when Sir2 is deleted, $\mathrm{CR}$ is not capable of lifespan extension (6). Furthermore, it has been demonstrated that in yeast, CR may activate Sir2 and consequently increase longevity by increasing respiration, since the deacetylase activity of Sir2 is regulated by the cellular oxidized nicotinamide adenine dinucleotide/reduced nicotinamide adenine dinucleotide $\left(\mathrm{NAD}^{+} / \mathrm{NADH}\right)$ ratio (6). It has been suggested that $\mathrm{CR}$ is capable of reducing the levels of NADH or the nicotinamide-degrading enzyme pyrazinamidase/nicotinamidase 1 (PNC1), which consequently activates the Sir2 deacetylase and increases the lifespan $(7,8)$. 
The Sir2 gene encodes a highly conserved NAD-dependent histone deacetylase (9). In mammals, there are seven homologues of Sir2 known as sirtuins (SIRT1-7). SIRTs have diverse cellular localizations, targeting multiple substrates and affecting a wide range of cellular functions, including the regulation of oxidative stress, DNA damage and metabolism (10). An increase in SIRT1 expression has been observed in numerous tissues of CR animals (11). However, the roles of SIRT2-7 during CR are yet to be elucidated. The present study investigated the cellular location of SIRTs in H9c2 cells and rat cardiac tissues, and is the first investigation, to the best of our knowledge, to demonstrate that short-term CR activates not only SIRT1 but also SIRT2-4 and -7 in cardiomyocytes in vivo and in vitro.

\section{Materials and methods}

Materials. A total of 20 three-month-old Sprague Dawley (SD) rats of both genders, weighing an average of $229.1 \mathrm{~g}$ each, were purchased from the Experimental Animal Centre of Shantou University Medical College (Shantou, China). Embryonic rat heart-derived H9c2 cells were obtained from the American Type Culture Collection (Rockville, MD, USA). Primary antibodies against SIRT1-5 and -7 and $\beta$-actin were purchased from Santa Cruz Biotechnology, Inc. (SIRT1, sc-15404; SIRT2, sc-20966; SIRT3, sc-49743; SIRT4, sc-66296; SIRT5, sc-66272; SIRT7, sc-66281, $\beta$-actin, sc-47778; Santa Cruz, CA, USA). The anti-SIRT6 antibody was purchased from Abcam (SIRT6, ab62739; Cambridge, MA, USA). An enhanced chemiluminescence (ECL) western blot detection system was obtained from GE Healthcare (Amersham, UK).

Animals and groups. SD rats were randomly divided into either a control group $(n=10)$ or a CR group $(n=10)$, with five males and five females in each group. Rats in the control group were fed ad libitum (AL), while rats in the CR group were fed at $60 \%$ of AL. Rats were housed individually. Sufficient vitamins and minerals were present in each of the diets, and the rats had unlimited access to water for three weeks. The components of the modified AL and CR diets are further described in Table I. Rats were monitored and weighed daily. All animal procedures were approved by the Animal Care and Use Committee of Shantou University Medical College.

Tissue processing. The rats $(n=20)$ were sacrificed after three weeks by cervical dislocation following anesthetisia. Hearts were harvested and flushed with $0.9 \%$ normal saline solution, prior to being sectioned into three parts. The central sections of each rat heart, which included dual atriums and dual ventricles, were used for immunohistochemical analysis. The two remaining sections were used for western blot analysis and were immediately stored at $-80^{\circ} \mathrm{C}$.

Immunohistochemical staining. The central heart sections were immersed in $4 \%$ parafomaldehyde for $24 \mathrm{~h}$. Serial transverse heart sections were deparaffinated in xylene and rehydrated in a graded ethanol series. Sections were pre-incubated with $0.3 \%$ hydrogen peroxide in phosphate-buffered saline (PBS) for $5 \mathrm{~min}$, in order to inactivate any endogenous peroxidase activity, prior to being blocked with $2 \%$ bovine serum albumin (BSA) for
$30 \mathrm{~min}$. Specimens were then incubated with primary antibodies against SIRT1-7 at a dilution of 1:25 (v/v), overnight at $4^{\circ} \mathrm{C}$. Sections were subsequently incubated with biotin-conjugated anti-rabbit or anti-goat immunoglobulin G (IgG) (Kirkegaard \& Perry Laboratories, Gaithersburg, MD, USA) at a dilution of $1: 100(\mathrm{v} / \mathrm{v})$ in PBS at room temperature for $1 \mathrm{~h}$, prior to the application of preformed avidin-biotin complex conjugated to peroxidase (Vector laboratories, Inc., Burlingame, CA. USA) for $30 \mathrm{~min}$. The bound complexes were visualized using the administration of a $0.05 \%$ solution of 3-3'-diaminobenzidine (DAB) and counterstained with Harris' hematoxylin. PBS was used instead of the primary antibody for negative control reactions.

Western blot analysis. Equal quantities of protein were extracted from the rat heart tissues according to standard protocols, prior to being separated using 12\% SDS-PAGE and then transferred to polyvinylidene fluoride membranes according to standard procedures. Following three washes with PBS, the membranes were soaked in 5\% nonfat dry milk for $2 \mathrm{~h}$ at room temperature and incubated with primary antibodies against $\beta$-actin or SIRT1-7 overnight at $4^{\circ} \mathrm{C}$. Membranes were then incubated with horseradish peroxidase-conjugated secondary antibodies for $1 \mathrm{~h}$ at room temperature. Immune complexes were subsequently visualized using ECL and the band intensities were measured and quantified using Quantity One ${ }^{\circledR}$ software (Bio-Rad Laboratories Inc., Berkley, CA, USA).

Cell culture. H9c2 cells were cultured in high-glucose Dulbecco's modified Eagle medium (DMEM) supplemented with $10 \%$ fetal bovine serum (FBS), $100 \mathrm{U} / \mathrm{ml}$ penicillin and $100 \mathrm{mg} / \mathrm{ml}$ streptomycin, in $5 \% \mathrm{CO}_{2}$ at $37^{\circ} \mathrm{C}$ for $48 \mathrm{~h}$. Cells in the control group (Con) were retained in DMEM containing $4.5 \mathrm{~g} / 1$ glucose, while the cells in the CR group were subsequently cultured in DMEM containing $1 \mathrm{~g} / 1$ glucose. Both groups were incubated in $5 \% \mathrm{CO}_{2}$ at $37^{\circ} \mathrm{C}$ for $24 \mathrm{~h}$.

Immunocytochemical staining. Semiquantitative analysis of $\mathrm{H} 9 \mathrm{c} 2$ cells was performed following cell seeding onto coverslips. Following washing, H9c2 cells were fixed using 4\% paraformaldehyde in PBS for 15 min and incubated with $5 \%$ Triton X-100 in PBS at room temperature for $20 \mathrm{~min}$. Cells were then blocked using 2\% BSA for $30 \mathrm{~min}$, prior to incubation with primary antibodies against SIRT1-7 at a dilution of 1:50 (v/v) overnight at $4^{\circ} \mathrm{C}$. Following multiple washes with PBS, slides were incubated with biotin-conjugated anti-rabbit or anti-goat immunoglobulin $\mathrm{G}$ at dilutions of 1:100 (v/v) for $1 \mathrm{~h}$ at room temperature. The slides were then incubated with the preformed avidin-biotin complex conjugated to peroxidase for $30 \mathrm{~min}$. The bound complexes were visualized using the application of a $0.05 \%$ solution of DAB, and counterstained with Harris' hematoxylin. PBS was used instead of the primary antibody for negative control reactions.

Quantitative polymerase chain reaction ( $q P C R)$. Total RNA was extracted from the H9c2 cells using the RNAsimple Total RNA Kit (Tiangen Biotech Co., Beijing, China) according to the manufacturer's instructions. First-strand cDNAs were synthesized by reverse transcription using oligos (dT) from RNA samples. The primers (Genecore Biotechnologies Co., Shanghai, China) used for PCR amplification are described 
Table I. Nutrient composition of AL and CR diets.

\begin{tabular}{lcc}
\hline Nutrient composition & AL diet (per $1 \mathrm{~g}$ diet) & CR diet (per 0.62 g diet) \\
\hline Protein $(\mathrm{g})$ & 0.19 & 0.19 \\
Carbohydrate (g) & 0.67 & 0.29 \\
Fat (g) & 0.04 & 0.04 \\
Vitamin mix (mg) & 9.48 & 9.48 \\
Mineral mix (mg) & 9.48 & 9.48 \\
Cholesterol (mg) & 0.20 & 0.20 \\
Cellulose (mg) & 47.38 & 47.38 \\
Dicalcium phosphate (mg) & 12.32 & 12.32 \\
Calcium carbonate (mg) & 5.21 & 5.21 \\
Potassium citrate (mg) & 15.64 & 15.64 \\
Choline bitartrate (mg) & 1.90 & 1.90 \\
Total calories & 3.80 & 2.28 \\
\hline
\end{tabular}

Compared with the animals maintained on the AL diet, CR animals consumed 57\% less carbohydrate, accounting for a $40 \%$ decrease in total caloric intake. Animals maintained on a CR diet consumed identical quantities of protein, fat, cholesterol and micronutrients to those maintained on the AL diet. CR, calorie restriction; AL, ad libitum.

Table II. Primer sequences used in quantitative polymerase chain reaction.

\begin{tabular}{lll}
\hline Gene & Forward sequences $\left(5^{\prime}-3 '\right)$ & Reverse sequences $\left(5^{\prime}-3^{\prime}\right)$ \\
\hline SIRT1 & CCAGATCCTCAAGCCATGT & TTGGATTCCTGCAACCTG \\
SIRT2 & TACCCAGAGGCCATCTTTGA & TGATGTGTGAAGGTGCCGT \\
SIRT3 & TACTTCCTTCGGCTGCTTCA & AAGGCG AAATCAGCCACA \\
SIRT4 & ACTGGGAGAAACTTGGGAAG & CTGGTGCACAAAGTCAACCT \\
SIRT5 & AGCAAGATCTGCCTCACCAT & GGATTTCCAGCAGGTTCTTG \\
SIRT6 & TTGTCAACCTGCAACCCA & GCTTGGGCTTATAGGAACCA \\
SIRT7 & TCTCTGAGCTCCATGGGAAT & CATGAGGAGCCGCATTACAT \\
18S rRNA & ATTCCGATAACGAACGAGAC & GGCATCACAGACCTGTTATTG
\end{tabular}

rRNA, ribosomal RNA; SIRT, sirtuin.

in Table II. The PCR conditions used for all primers were as follows: DNA denaturation at $94^{\circ} \mathrm{C}$ for $30 \mathrm{sec}, 30$ cycles of $94^{\circ} \mathrm{C}$ for $30 \mathrm{sec}, 56^{\circ} \mathrm{C}$ for $30 \mathrm{sec}$ and $72^{\circ} \mathrm{C}$ for $60 \mathrm{sec}$, followed by a final extension step at $72^{\circ} \mathrm{C}$ for $6 \mathrm{~min}$. PCR products were electrophoresed in $2 \%$ agarose gels and visualized using ethidium bromide. Relative mRNA expression was quantified densitometrically using the Gel Image System version 3.74 (Tianon, Shanghai, China).

Statistical analysis. All data are presented as the mean \pm standard deviation of at least three independent experiments. Statistical analyzes were performed using one-way analysis of variance and t-tests with a correction for multiple comparisons where appropriate. A P-value of $<0.05$ was considered to indicate a statistically significant difference.

\section{Results}

$C R$ rats exhibit significantly lower $B W$ and heart weight. No significant differences were identified in BW of male (M) or female $(\mathrm{F})$ rats between the AL and CR groups prior to the treatment conditions of the present study [AL F: $230.20 \pm 16.08 \mathrm{~g}$ $(n=5)$ vs. CR F: $219.20 \pm 5.63 \mathrm{~g}(\mathrm{n}=5) ;$ AL M: $236.00 \pm 22.19 \mathrm{~g}$ $(\mathrm{n}=5)$ vs. CR M: $231.00 \pm 10.89 \mathrm{~g}(\mathrm{n}=5)]$. Following three weeks on either the CR or AL diets, BW was significantly higher in the AL group than in the CR group [AL F: 302.60 $\pm 34.60 \mathrm{~g}(\mathrm{n}=5)$ vs. CR F: $243.00 \pm 8.60 \mathrm{~g}(\mathrm{n}=5 ; \mathrm{P}<0.05)$; AL M: $292.20 \pm 34.85 \mathrm{~g}$ $(\mathrm{n}=5)$ vs. CR M: $253.40 \pm 3.78 \mathrm{~g}(\mathrm{n}=5, \mathrm{P}<0.05)]$ (Fig. 1A). The heart mass index (HMI=Heart weight/BW) was also significantly higher in the AL group [AL F: $2.90 \pm 0.06 \mathrm{mg} / \mathrm{g}$ $(\mathrm{n}=5)$ vs. CR F: $2.72 \pm 0.11 \mathrm{mg} / \mathrm{g} \quad(\mathrm{n}=5, \mathrm{P}<0.05)$ and AL M: $2.89 \pm 0.11 \mathrm{mg} / \mathrm{g}(\mathrm{n}=5)$ vs. CR M: $2.75 \pm 0.06 \mathrm{mg} / \mathrm{g}(\mathrm{n}=5$, $\mathrm{P}<0.05)$ ] (Fig. 1B).

Cellular localization of SIRT1-7 in rat cardiac tissues. SIRTs demonstrate diverse cellular locations and various cellular functions. Using immunohistochemistry, seven SIRT proteins were detected in the cardiac tissues of SD rats in the AL and CR groups (Fig. 2A). SIRT1 was observed to be distributed throughout the nucleus and the cytoplasm, whereas SIRT2-5 
A

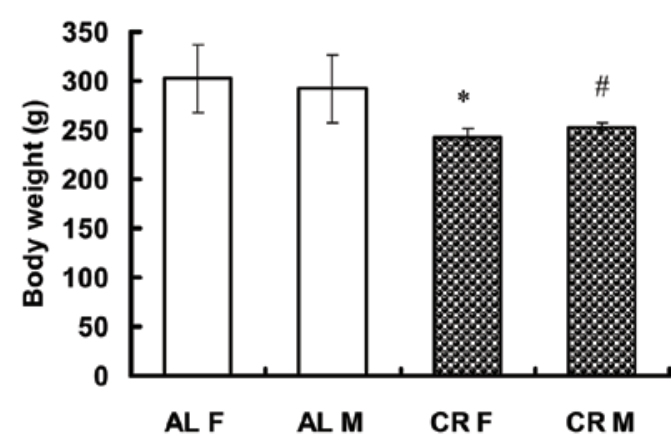

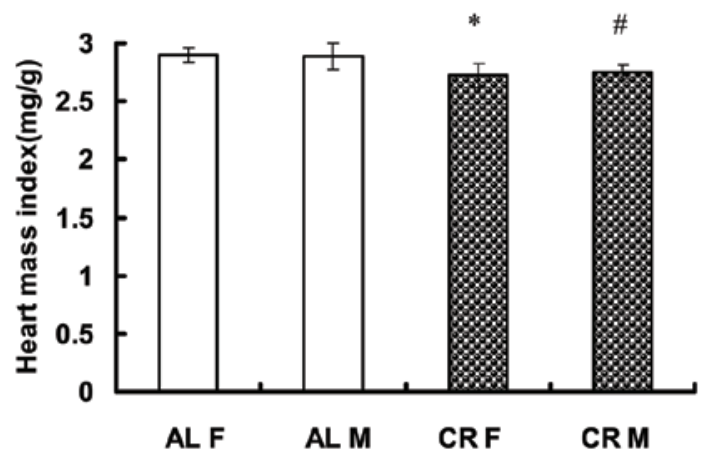

Figure 1. Changes in the BW and HMI (heart weight/BW) following maintenance on a CR diet for three weeks. CR rats show (A) a significantly lower BW and (B) a significantly lower heart weight compared with AL rats . ${ }^{*} \mathrm{P}<0.05$ vs. AL F group; ${ }^{\text {P }<0.05 ~ v s . ~ A L ~ M ~ g r o u p . ~ B W, ~ b o d y ~ w e i g h t ; ~ H M I, ~ h e a r t ~ m a s s ~ i n d e x ; ~}$ $\mathrm{AL}$, ad libitum; CR, calorie restriction; F, female, $\mathrm{M}$, male.

A
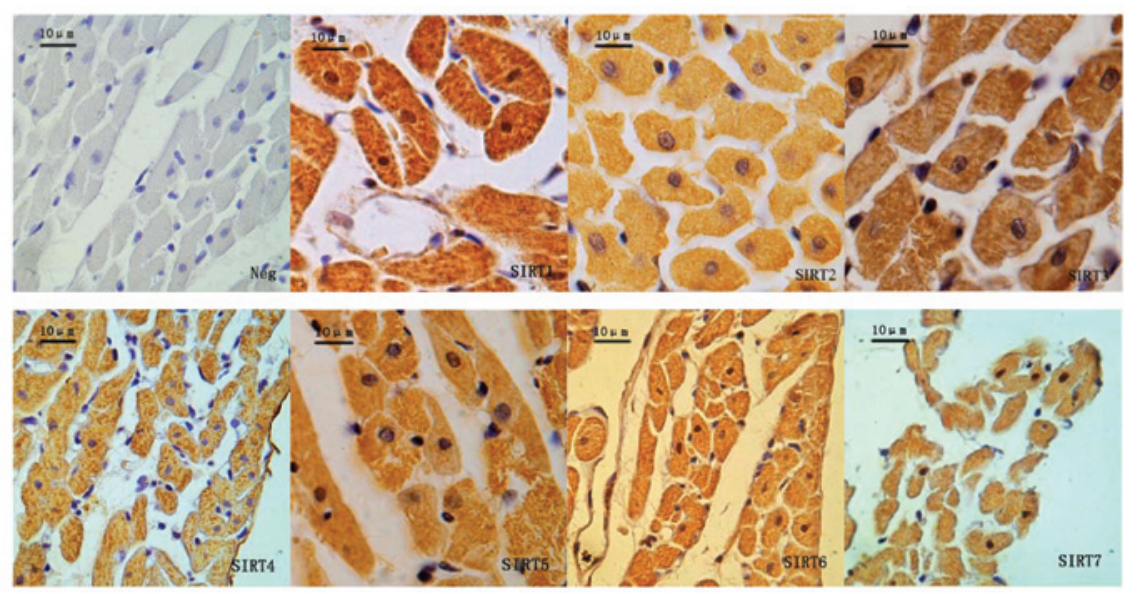

B
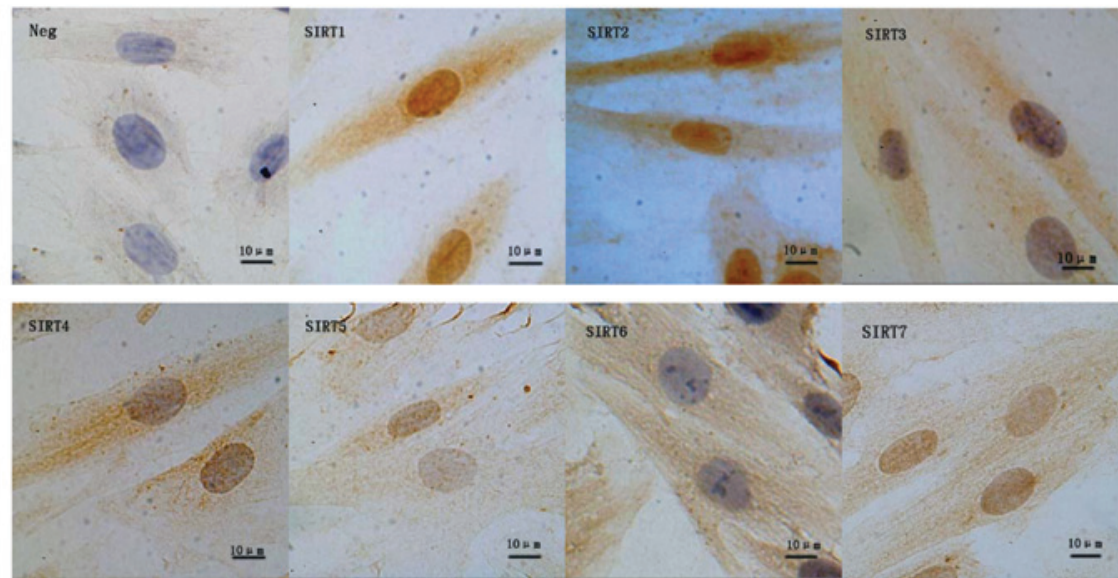

Figure 2. Cellular localization of SIRT1-7 in rat cardiac tissue and H9c2 cells. (A) Immunohistochemistry of the cellular localization of SIRT1-7 in rat cardiac tissues. (B) Immunocytochemistry of the cellular localization of SIRT1-7 in H9c2 cells. A similar pattern of SIRT1, 3-5 and -7 localization was observed in H9c2 cells and rat cardiac tissues. SIRT1 and -7 were found predominantly in the nucleolus, while SIRT3-5 were found predominantly in the cytoplasm. SIRT2 was detected exclusively in the cytoplasm in rat cardiac tissues; however, it was found predominantly in the nucleolus in H9c2 cells, while the contrary was observed for SIRT6. Magnification, x1,000. SIRT, sirtuin.

were detected primarily in the cytoplasm, and SIRT6 and -7 predominantly in the nucleus.

Cellular localization of SIRT1-7 in H9c2 cells. Immunocytochemistryrevealed that SIRT1, 3-5 and -7 exhibited a similar pattern of distribution in $\mathrm{H} 9 \mathrm{c} 2$ cells as in rat heart tissues (Fig. 2B). SIRT1 and 2 were identified in the nucleus and cytoplasm; however, SIRT3-6 were detected predominantly in the cytoplasm and SIRT7 in the nucleus.

CR upregulates the protein expression of SIRT1-4 and -7 in rat cardiac tissues. To investigate the effect of CR on SIRT 
A

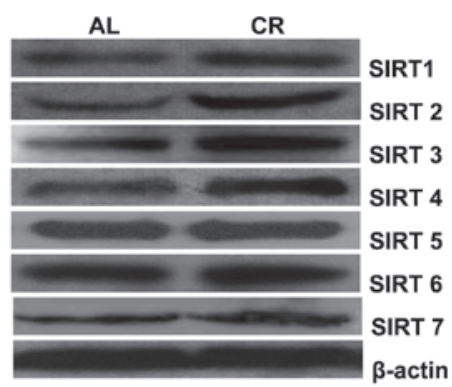

B

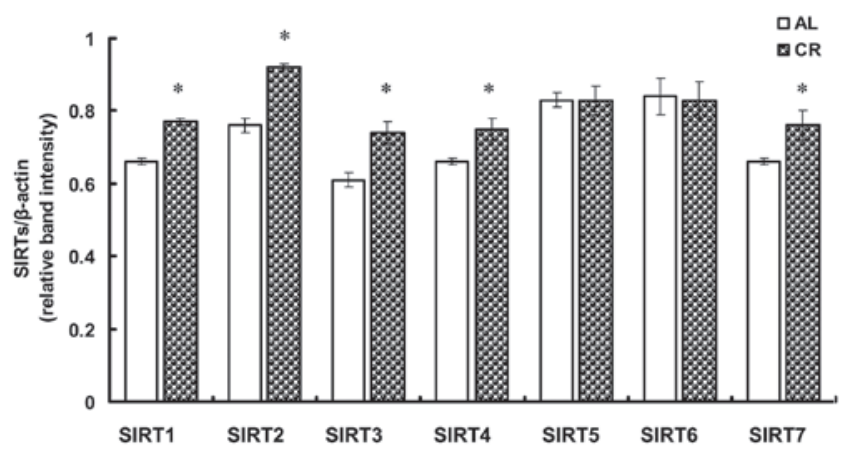

Figure 3. Western blot analysis of SIRT1-7 protein expression in the heart tissues of SD rats. Twenty SD rats were maintained on either AL or CR $(60 \%$ of AL) diets for three weeks. (A) Western blot analysis showing SIRT1-7 and $\beta$-actin protein expression in the same sample. (B) SIRT1-7 protein abundance normalized to $\beta$-actin expression. Values are expressed as the mean \pm standard deviation for three independent experiments. ${ }^{*} \mathrm{P}<0.05$ vs AL. SD, Sprague Dawley; AL, ad libitum; CR, calorie restriction; SIRT, sirtuin.

expression in rat hearts, 20 three-month-old SD rats were fed either AL or a CR diet for three weeks. The changes in SIRT1-7 protein expression were subsequently analyzed. Western blot analysis revealed that SIRT1-4 and -7 expression were significantly upregulated in the $\mathrm{CR}$ group compared with the AL group $(\mathrm{P}<0.05)$; however, no significant difference was observed in the expression of SIRT5 and 6 (Fig. 3).

CR upregulates the mRNA expression of SIRT1-4 and -7 in $H 9 c 2$ cells. To support the results mentioned previously, $\mathrm{H} 9 \mathrm{c} 2$ cells were cultured in either normal $(4.5 \mathrm{~g} / \mathrm{l})$ or low $(1 \mathrm{~g} / \mathrm{l})$ concentrations of glucose for $24 \mathrm{~h}$, representing control and CR groups, respectively. qPCR analysis revealed that the mRNA expression of SIRT1-4 and 7 was significantly higher in the $\mathrm{CR}$ group compared with the control group $(\mathrm{P}<0.05)$; however, SIRT5 and 6 mRNA expression did not differ significantly (Fig. 4).

\section{Discussion}

CR has been observed to markedly extend the lifespan in a wide range of organisms and attenuate numerous age-associated diseases, i.e. CVD, partially through metabolic mechanisms, including decreasing BW, body fat, blood glucose, insulin, triglyceride and cholesterol, and increasing insulin sensitivity and glucose tolerance (12). Furthermore, CR has been demonstrated to directly affect the cardiovascular system, improving the function of endothelial and smooth muscle cells (13). Moreover, long-term CR has been found to attenuate age-associated diastolic dysfunction and ischemic damage in
$\mathbf{A}$

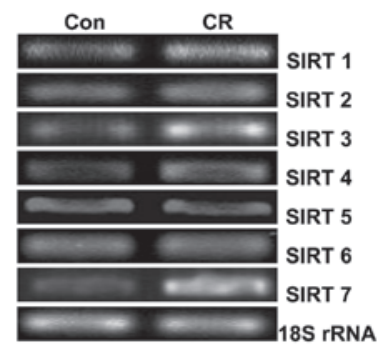

B

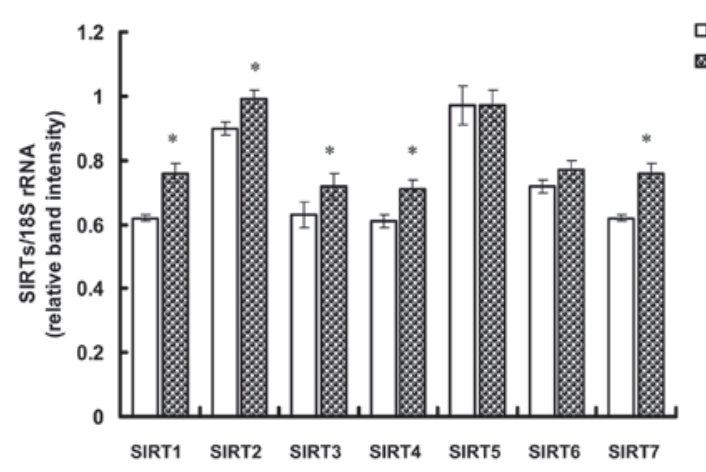

Figure 4. SIRT1-7 mRNA expression in H9c2 cells using quantitative polymerase chain reaction analysis. H9c2 cells were grown for $24 \mathrm{~h}$ in Dulbecco's modified Eagle medium supplemented with either normal glucose concentrations (4.5 g/1, Con) or low glucose concentrations (1 g/l, CR). (A) Ethidium bromide-stained gels of target products and 18S rRNA in the same sample. (B) SIRT1-7 mRNA abundance normalized to $18 \mathrm{~S}$ rRNA expression. The results are presented as the mean \pm standard deviation of three independent experiments. ${ }^{*} \mathrm{P}<0.05$ vs. Con. SIRT, sirtuin; Con, control; CR, calorie restriction; rRNA, ribosomal RNA.

an experimental model of myocardial infarction in rats $(14,15)$. It was observed in the present study that short-term $\mathrm{CR}$ reduced heart weight and $\mathrm{BW}$ in rats, consistent with the metabolic effects of CR.

Evidence suggests that Sir2 is a significant regulator of responses to CR (2). Sir2 is a highly conserved NAD-dependent histone deacetylase that has been shown to modulate lifespan in numerous species $(9,16)$. Seven members of the Sir2 family, known as SIRTs (SIRT1-7), exist in mammals, among which SIRT1 is the closest homologue of the yeast Sir2 protein $(17,18)$. In addition to histone deacetylation, SIRT1 is capable of deacetylating other proteins, including Forkhead transcription factors (FoxOs), myogenic differentiation antigen (MyoD), peroxisome proliferator-activated receptor- $\gamma$ coactivator (PGC)-1 $\alpha$ and the tumor suppressor p53 (19-24). SIRT1 is therefore capable of regulating cellular metabolism and exerting corresponding effects on gene expression. SIRT1 is a key regulator of cellular defense mechanisms and survival under stress $(11,21,22,25,26)$. Furthermore, SIRT1 has been observed to improve vasodilatory and regenerative functions in endothelial and smooth muscle cells of the vascular wall, through regulation of the activity of endothelial nitric oxide synthase, FoxO1, p53 and angiotensin II type 1 receptor (AT1R), and is therefore suggested to have a cardioprotective role (27).

SIRTs demonstrate diverse cellular localizations and numerous cellular functions. SIRT1 is located in the nucleus and cytoplasm in cardiomyocytes; however, its FoxO deacetylation activty is restricted to the nucleus, where it is capable of forming a protein complex with 
FoxOs $(28,29)$. The cellular localizations of SIRT2-7 in cardiomyocytes are unknown. The present study examined the cellular localization of SIRT1-7 in H9c2 cells and rat cardiac tissue. Consistent with a previous study (30), SIRT1 was identified in the nucleus and cytoplasm, while SIRT7 was predominantly observed in the nucleus. SIRT3-5 were detected primarily in the cytoplasm, in accordance with previous findings $(30,31)$. Significantly, the present study identified that in the cardiac tissues of SD rats, SIRT6 was predominantly identified in the nucleus, in accordance with a previous report (32); however, a high concentration of SIRT6 was detected in the cytoplasm in $\mathrm{H} 9 \mathrm{c} 2$ cells. The embryonic nature of $\mathrm{H} 9 \mathrm{c} 2$ cells is hypothesized to be responsible for this discrepancy. Contrary results were obtained for SIRT2.

It is well established that SIRTs are upregulated by CR. Cohen et al (11) suggested that in response to CR, SIRT1 is upregulated in numerous tissues, including the brain, visceral fat pads, kidney and liver. Furthermore, the expression of SIRT2 has been observed to increase in the white adipose tissue and kidneys of mice in response to CR (33). Moreover, Shi et al (34) demonstrated that following three months of CR, SIRT3 mRNA levels were elevated in both white and brown adipose tissue in male mice. However, CR treatment has been observed to decrease the expression of SIRT4 in pancreatic $\beta$-cells $(35,36)$. The roles of SIRT1-7 in CR responses in cardiomyocytes are yet to be elucidated; however, the present study revealed that CR significantly upregulated SIRT1-4 and 7 mRNA and protein levels, suggesting that endogenous SIRT1-4 and -7 have a significant role in CR responses in cardiomyocytes. The results obtained for SIRT4 were inconsistent with the findings of Mahlknecht and Voelter-Mahlknecht (35) and Chen et al (36), which may be a consequence of the different cell lines employed.

In conclusion, the present study has identified the presence of seven SIRTs in SD rat cardiac tissues and in H9c2 cells, and has demonstrated that endogenous SIRT1-4 and -7 may participate, or have an essential role, in mediating CR responses in cardiomyocytes in vivo and in vitro. These findings may be of significance for attenuating age-associated diseases, particularly CVD. Modulating SIRT1-4 and -7 activities is likely to achieve similar biological effects to CR diet treatment. These findings may aid the development of novel therapies for the treatment of CVD, including drugs or biological methods that activate SIRTs. Such treatments are anticipated to deliver broad benefits for CVD.

\section{Acknowledgements}

The present study was supported by the Natural Science Foundation of China (no. 81270382) and the Natural Science Foundation of Guangdong Province, P.R. China (no. 10151503102000039).

\section{References}

1. McCay CM, Crowell MF and Maynard LA: The effect of retarded growth upon the length of life span and upon the ultimate body size. 1935. Nutrition 5: 155-172, 1989 .

2. Lin SJ, Defossez PA and Guarente L: Requirement of NAD and SIR2 for life-span extension by calorie restriction in Saccharomyces cerevisiae. Science 289: 2126-2128, 2000.
3. Ingram DK, Zhu M, Mamczarz J, Zou S, Lane MA, Roth GS and deCabo R: Calorie restriction mimetics: an emerging research field. Aging Cell 5: 97-108, 2006.

4. Bordone L and Guarente L: Calorie restriction, SIRT1 and metabolism: understanding longevity. Nat Rev Mol Cell Biol 6: 298-305, 2005.

5. Ungvari Z, Parrado-Fernandez C, Csiszar A and de Cabo R: Mechanisms underlying caloric restriction and lifespan regulation: implications for vascular aging. Circ Res 102: 519-528, 2008.

6. Lin SJ, Kaeberlein M, Andalis AA, Sturtz LA, Defossez PA, Culotta VC, Fink GR and Guarente L: Calorie restriction extends Saccharomyces cerevisiae lifespan by increasing respiration. Nature 418: 344-348, 2002.

7. Anderson RM, Bitterman KJ, Wood JG, Medvedik O and Sinclair DA: Nicotinamide and PNC1 govern lifespan extension by calorie restriction in Saccharomyces cerevisiae. Nature 423: 181-185, 2003.

8. Lin SJ, Ford E, Haigis M, Liszt G and Guarente L: Calorie restriction extends yeast life span by lowering the level of NADH. Genes Dev 18: 12-16, 2004.

9. Imai S, Armstrong CM, Kaeberlein M and Guarente L: Transcriptional silencing and longevity protein Sir2 is an NAD-dependent histone deacetylase. Nature 403: 795-800, 2000.

10. Michan S and Sinclair D: Sirtuins in mammals: insights into their biological function. Biochem J 404: 1-13, 2007.

11. Cohen HY, Miller C, Bitterman KJ, Wall NR, Hekking B, Kessler B, Howitz KT, Gorospe M, de Cabo R and Sinclair DA: Calorie restriction promotes mammalian cell survival by inducing the SIRT1 deacetylase. Science 305: 390-392, 2004

12. Koubova J and Guarente L: How does calorie restriction work? Genes Dev 17: 313-321, 2003.

13. Mattagajasingh I, Kim CS, Naqvi A, Yamamori T, Hoffman TA, Jung SB, DeRicco J, Kasuno K and Irani K: SIRT1 promotes endothelium-dependent vascular relaxation by activating endothelial nitric oxide synthase. Proc Natl Acad Sci USA 104: 14855-14860, 2007.

14. Shinmura K, Tamaki K, Sano M,Murata M, Yamakawa H,IshidaH and Fukuda K: Impact of long-term caloric restriction on cardiac senescence: caloric restriction ameliorates cardiac diastolic dysfunction associated with aging. J Mol Cell Cardiol 50: 117-127, 2011.

15. Ahmet I, Tae HJ, de Cabo R, Lakatta EG and Talan MI: Effects of calorie restriction on cardioprotection and cardiovascular health. J Mol Cell Cardiol 51: 263-271, 2011.

16. Smith JS, Brachmann CB, Celic I, Kenna MA, Muhammad S, Starai VJ, Avalos JL, Escalante-Semerena JC, Grubmeyer C, Wolberger C and Boeke JD: A phylogenetically conserved $\mathrm{NAD}^{+}$-dependent protein deacetylase activity in the Sir2 protein family. Proc Natl Acad Sci USA 97: 6658-6663, 2000.

17. Frye RA: Characterization of five human cDNAs with homology to the yeast SIR2 gene: Sir2-like proteins (sirtuins) metabolize NAD and may have protein ADP-ribosyltransferase activity. Biochem Biophys Res Commun 260: 273-279, 1999.

18. Frye RA: Phylogenetic classification of prokaryotic and eukaryotic Sir2-like proteins. Biochem Biophys Res Commun 273: 793-798, 2000.

19. Luo J, Nikolaev AY, Imai S, Chen D, Su F, Shiloh A, Guarente L and Gu W: Negative control of p53 by Sir2alpha promotes cell survival under stress. Cell 107: 137-148, 2001.

20. Fulco M, Schiltz RL, Iezzi S, King MT, Zhao P, Kashiwaya Y, Hoffman E, Veech RL and Sartorelli V: Sir2 regulates skeletal muscle differentiation as a potential sensor of the redox state. Mol Cell 12: 51-62, 2003.

21. Brunet A, Sweeney LB, Sturgill JF, et al: Stress-dependent regulation of FOXO transcription factors by the SIRT1 deacetylase. Science 303: 2011-2015, 2004.

22. Motta MC, Divecha N, Lemieux M, Kamel C, Chen D, Gu W, Bultsma Y, McBurney M and Guarente L: Mammalian SIRT1 represses forkhead transcription factors. Cell 116: 551-563, 2004.

23. Rodgers JT, Lerin C, Haas W, Gygi SP, Spiegelman BM and Puigserver P: Nutrient control of glucose homeostasis through a complex of PGC-1alpha and SIRT1. Nature 434: 113-118, 2005.

24. Cao C, Lu, S, Kivlin R, Wallin B, et al: SIRT1 confers protection against UVB- and $\mathrm{H}_{2} \mathrm{O}_{2}$-induced cell death via modulation of p53 and JNK in cultured skin keratinocytes. J Cell Mol Med 13: 3632-3643, 2009.

25. Vaziri H, Dessain SK, Ng Eaton E, Imai SI, Frye RA, Pandita TK, Guarente L and Weinberg RA: hSIR2(SIRT1) functions as an NAD-dependent p53 deacetylase. Cell 107: 149-159, 2001. 
26. Kobayashi Y, Furukawa-Hibi Y, Chen C, Horio Y, Isobe K, Ikeda K and Motoyama N: SIRT1 is critical regulator of FOXO-mediated transcription in response to oxidative stress. Int J Mol Med 16: 237-243, 2005.

27. Borradaile NM and Pickering JG: NAD(+), sirtuins, and cardiovascular disease. Curr Pharm Des 15: 110-117, 2009.

28. Wang Y and Tissenbaum HA: Overlapping and distinct functions for a Caenorhabditis elegans SIR2 and DAF-16/FOXO. Mech Ageing Dev 127: 48-56, 2006.

29. Chen CJ, Yu W, Fu YC, Wang X, Li JL and Wang W: Resveratrol protects cardiomyocytes from hypoxia-induced apoptosis through the SIRT1-FoxO1 pathway. Biochem Biophys Res Commun 378: 389-393, 2009

30. Michishita E, Park JY, Burneskis JM, Barrett JC and Horikawa I: Evolutionarily conserved and nonconserved cellular localizations and functions of human SIRT proteins. Mol Biol Cell 16: 4623-4635, 2005.

31. North BJ, Marshall BL, Borra MT, Denu JM and Verdin E: The human Sir2 ortholog, SIRT2, is an $\mathrm{NAD}^{+}$-dependent tubulin deacetylase. Mol Cell 11: 437-444, 2003.
32. Liszt G, Ford E, Kurtev M and Guarente L: Mouse Sir2 homolog SIRT6 is a nuclear ADP-ribosyltransferase. J Biol Chem 280: 21313-21320, 2005.

33. Wang F, Nguyen M, Qin FX and Tong Q: SIRT2 deacetylates FOXO3a in response to oxidative stress and caloric restriction. Aging Cell 6: 505-514, 2007.

34. Shi T, Wang F, Stieren E and Tong Q: SIRT3, a mitochondrial sirtuin deacetylase, regulates mitochondrial function and thermogenesis in brown adipocytes. J Biol Chem 280: 13560-13567, 2005.

35. Mahlknecht U and Voelter-Mahlknecht S: Fluorescence in situ hybridization and chromosomal organization of the sirtuin 4 gene (Sirt4) in the mouse. Biochem Biophys Res Commun 382: 685-690, 2009.

36. Chen YR, Fang SR, Fu YC, Zhou XH, Xu MY and Xu WC: Calorie restriction on insulin resistance and expression of SIRT1 and SIRT4 in rats. Biochem Cell Biol 88: 715-722, 2010. 